\title{
DISPARITY MAP GENERATION BASED ON TRAPEZOIDAL CAMERA ARCHITECTURE FOR MULTI-VIEW VIDEO
}

\author{
Abdulkadir Iyyaka Audu and Abdul Hamid Sadka \\ Department of Electronic and Computer Engineering, \\ Brunel University, London, United Kingdom
}

\begin{abstract}
Visual content acquisition is a strategic functional block of any visual system. Despite its wide possibilities, the arrangement of cameras for the acquisition of good quality visual content for use in multi-view video remains a huge challenge. This paper presents the mathematical description of trapezoidal camera architecture and relationships which facilitate the determination of camera position for visual content acquisition in multi-view video, and depth map generation. The strong point of Trapezoidal Camera Architecture is that it allows for adaptive camera topology by which points within the scene, especially the occluded ones can be optically and geometrically viewed from several different viewpoints either on the edge of the trapezoid or inside it. The concept of maximum independent set, trapezoid characteristics, and the fact that the positions of cameras (with the exception of few) differ in their vertical coordinate description could very well be used to address the issue of occlusion which continues to be a major problem in computer vision with regards to the generation of depth map.
\end{abstract}

\section{KEYWORDS}

Multi-view Video, Occlusion, Stereoscopic distance, Trapezoidal Camera Architecture, Visual Content Acquisition

\section{INTRODUCTION}

Human vision system (HVS) has stimulated an interest in acquisition of visual content and provided an acceptable guide to its understanding. The subject of visual content acquisition developed very early in history because its principles can be formulated from HVS of which visual attention forms an important characteristic. Right from the very beginning it promises a photorealistic replica of scenes for enormous emerging domains with many applications in many different areas. In this regard, mathematical descriptions of propagation pattern of light, parameterization of the behavioral response of optical devices are required.

A substantial body of evidence according to [1] demonstrates that exposure experience allows the individual to learn a great deal about the stimulus object, so that the ability to recognize, discriminate, and categorize the object generally improves. The first order step in the achievement of these improvements is the registration of both optical and geometric properties of 
The International Journal of Multimedia \& Its Applications (IJMA) Vol.6, No.6, December 2014

the scene. The identification of this knowledge has triggered the visual content acquisition of scenes from a single viewpoint using optical cameras.

On the one hand, the simplest image acquisition is a two-dimensional (2D) array of light intensity functions representing points in the three-dimensional (3D) world. The intensity function at each spatial coordinate is determined by the degree of illumination at that coordinate. The uniqueness and potential of information embedded in 2D image has brought about the convergence of fields such as image processing, computer graphics, pattern recognition, artificial intelligence, psychophysics, and machine vision [1]. On the other hand, a complete description of an object in terms of its physical shape and size is only possible when 3D coordinates are available. 3D acquisition requires a geometrically and photometrically calibrated camera.

Building on the premise of distributed vision-based sensing and processing, multi-camera networks have emerged as one of the preferred approaches for generating 3D content. 3D content is known to provide depth perception of the observed scene [2]. Such content can be obtained by having a high-resolution, wide-angle camera focused during a moderate object motion [3]. However, in computer vision, a synchronized set of multi-cameras with known accurate positions and orientations, brightness and chromatic characteristics are used to observe object surface areas. Single camera techniques, Holoscopic capture devices, pattern projection technique, and time-of-flight techniques have been actively used in 3D content acquisition in other applications.

One important application of multi-camera system is in multi-view video. This is popularly used in free viewpoint television (FVT), [4], [5], which enables a 3D scene to be viewed by freely changing our viewpoint, and 3D television (3DTV) in which the illusion of depth is created. Multi-view imaging has also attracted increasing attention in another wide variety of interesting new research topics and applications. These range from virtual view synthesis, high performance imaging, image and video segmentation, object tracking and recognition, environmental surveillance, remote education, to industrial inspection [6]. Its application in video content service such as video summarization [7], a condensed form of video content is generated for the purpose of browsing, retrieval and storage enhancement. 3D seismology now has a considerable driving force as opposed to its two-dimensional counterpart. It can help to solve the increased dimensionality of the problems associated with imaging, processing, and visualization of resultant images.

Until recently, however, the focus has been on understanding the brilliant successes of three lines of development in camera configurations such as parallel array, convergence array, and divergence array, Figure 1. Parallel array is the simplest form in which identical cameras are all in a linear orientation. It is mentioned in [8] that more complicated settings can have different camera lens properties and zoom facing the same 3D scene from different directions. The geometry of these camera topologies can very easily be analysed. The need to improve the camera architecture used in visual acquisition has been made evident from a variety of angles. This includes the issue of camera density, the reduction in the number of physical cameras, image quality, synchronization [9], depth estimation and occlusion [10], [11]. 


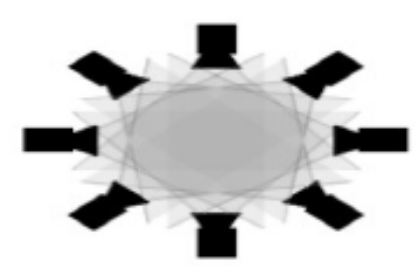

a

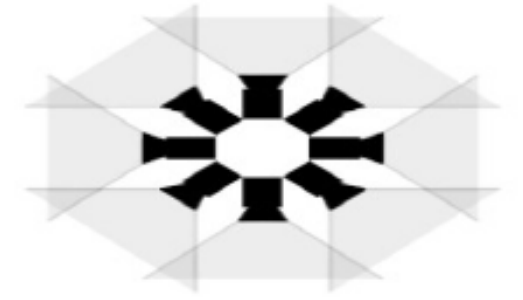

$\mathrm{b}$

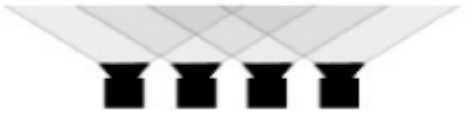

C

Figure 1 Multi-view camera arrangements: (a) converging, (b) diverging, and (c) parallel arrangement

Fairly recently, in [12], other issues such as visual attention has been considered as an important aspect of perception and its understanding is therefore necessary in the creation of 3D content. Reference [13] has targeted an important discussion at remapping the disparity range of stereoscopic images and video. This is aimed at reducing the effect of a complex combination of perceptual, technological, and artistic constraints on the displayed depth and the resulting 3D viewing experience. A promising approach to measure $3 \mathrm{D}$ visual fatigue using electroencephalogram and event-related potential has been proposed by [14].

The challenge of camera placement in multi-camera setting has been highlighted in [15]. Optimum multi-camera configuration is formulated based on a given volumetric space, placement constraint, and cost function. The solution to this optimization problem is sought from the point of view of bee colony algorithm. Unfortunately, in the acquisition of visual content, the problem remains to find a good arrangement of cameras which allows for the generation of quality content.

In this paper, the mathematical description of a remarkable multi-camera topology: the trapezoidal camera architecture (TCA), for acquisition of visual content is proposed. This is in spite of the fact that best observability of the object surface with a single ring camera arrangement can be achieved when the ring is at mid-height of the target object [3]. The strong point of TCA is that it is based on an efficient trapezoid which is half of a regular hexagon. More importantly, a trapezoid defines four of the six sides of a frustum (hexahedral) which has become an interesting topic of intense research in mesh generation. In [16], it is confirmed that quadrilateral and hexahedral elements are useful for finite elements and finite volume methods for mesh generation, and for some applications they are preferred to triangles or tetrahedral. A trapezoid is a quadrilateral with a pair (or at least one) of opposite parallel sides [17]. Acquisition cameras can be arranged on the sides of a trapezoid as shown in Figure 2. Though both conceptual and implementation challenges are in view, the architecture of Figure 2 can be implemented at a certain frequency around a scene as depicted in Figure 3.

TCA could facilitate a change in the emphasis for the realization of virtual camera. Of course, this computational photography realized in software provides a unique way to reduce the number of physical cameras used in the acquisition of visual content. A critical concern is the determination of the stereoscopic distance between a virtual viewpoint and any physical camera around it. 
The International Journal of Multimedia \& Its Applications (IJMA) Vol.6, No.6, December 2014

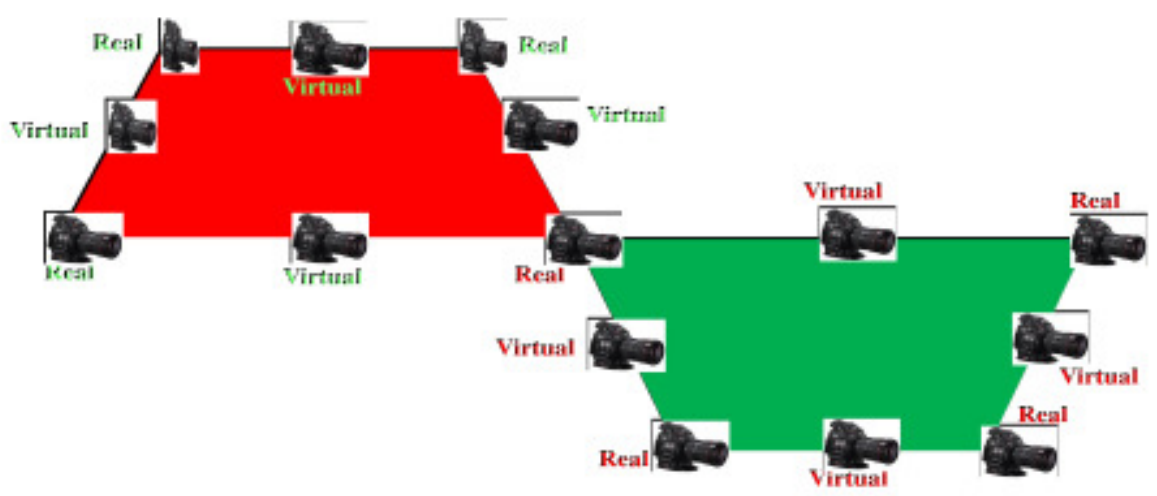

Figure 2. Trapezoidal camera architecture

Also the analysis of TCA boils down to a search problem which entails the determination of maximum independent set (MIS). Given a trapezoid graph, the number of integer vertices for which no two have an edge between them and no additional vertex can be added is known as MIS. Since all the cameras are not at the same horizontal level, the problem of occlusion has a great chance of being addressed.

It provides an exponential population of possible viewpoints from which any points within the scene can be seen. This approach has the tendency to dramatically address the issue of occluded parts of an object even though the technique of synthetic aperture focusing is a design space that has been explored in [18]. Therefore the potential of TCA is explored in the generation of high quality depth map with respect to different virtual viewpoints. Significantly, depth map is one of the requirements for view synthesis in depth-image-based rendering (DIBR).

It is worth noting that the trigonometric, area, sides and distances, and collinearity characteristics of this architecture very easily allow the baseline separation between any two cameras to be accurately computed. The relevance of this parameter is enormous since it is the sole determinant in the categorization of camera topology as either dense or sparse.

TCA is worth considering since a strong argument [19] has been put forward in favour of radially captured images. It is stated that linear array of cameras is considered to be the more correct method of capturing stereo images and does not suffer from distortion. However, convergence camera arrangement is simpler to implement: standard cameras or renderers can be used with no modification. Also, with respect to depth map acquisition, optimization of matching energy function defined by Markov Random Field (MRF) can now be optimized. This efficiently provides for rectification of the image pairs acquired through convergence camera array [20].

One eminent merit which triggers a very strong line of discussion in support of TCA is driven by the concept on which concentric mosaicking is based, as depicted in Figure 4. If a camera is rotated anti-clockwise along the circumference of a circle, a corresponding sinusoidal path, whose frequency depends on the speed of the camera, is generated. It therefore means that the reverse process whereby a camera translates along a sinusoidal path should be capable of realizing a concentric mosaic. Since an efficient trapezoid is a truncated or clipped sinusoid, it does intellectually make sense in the face of conceptual and implementation challenges, to be able to use a set of cameras arranged on the perimeter of a trapezoid to capture a scene in multiview video. 
The International Journal of Multimedia \& Its Applications (IJMA) Vol.6, No.6, December 2014

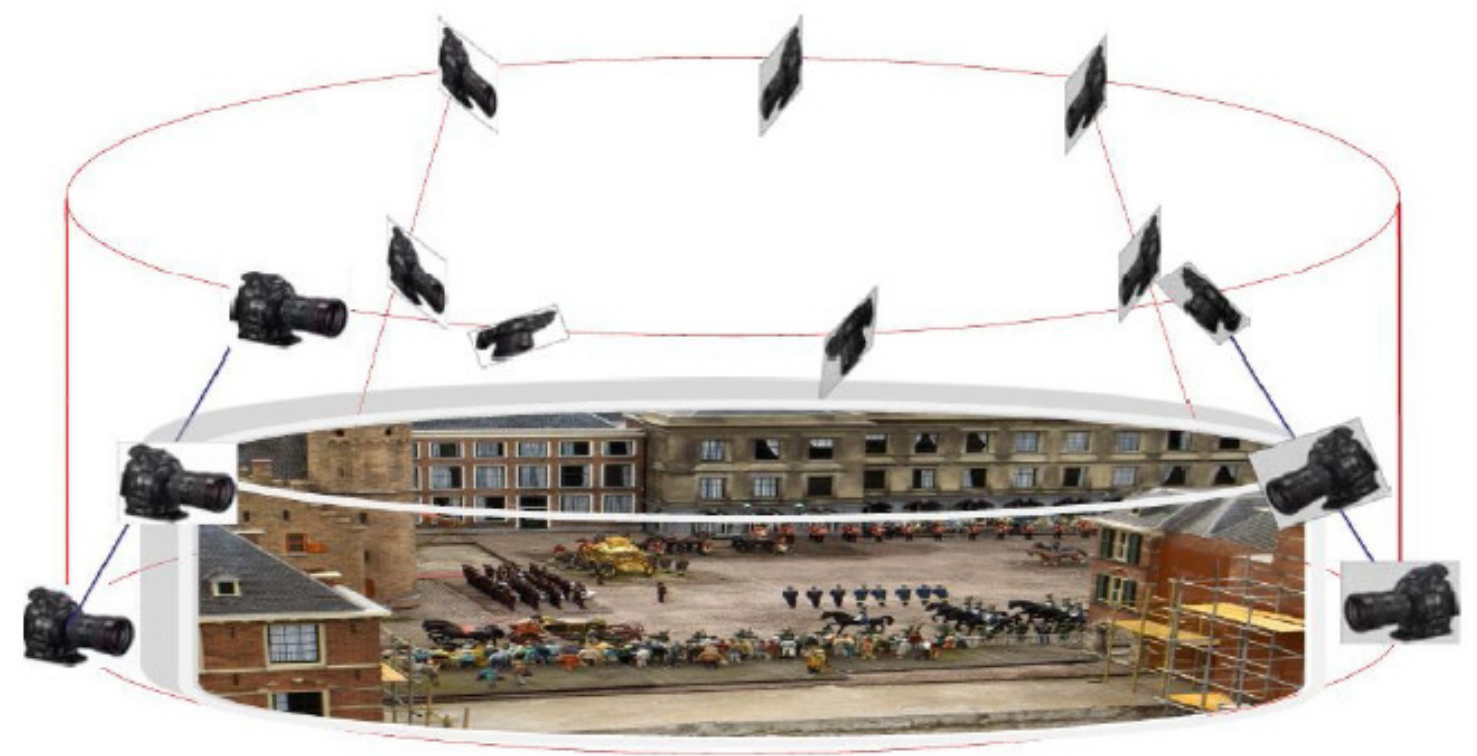

Figure 3. Pictorial representation of scene and trapezoidal camera architecture

The other discussions in this paper will unfold as follows. Section II will present a review of already known camera architectures. A comprehensive explanation of TCA will be given in section III. In section IV, conceptual and implementation challenges are highlighted. The conclusion of this work is drawn in section $\mathrm{V}$.

\section{Conventional Camera Architecture}

A brief review of the existing camera architectures namely : parallel, convergence, and divergence, is presented in this section.

\subsection{Parallel array}

According to [21] parallel arrangement of cameras allows for wide angle capturing of the scene. It is also known to feature simple disparity calculation. A hybrid camera system consisting of five high-definition video cameras arranged in a linear array and one time-of-flight depth camera for the generation of multi-view video has been proposed by [22]. The merit of this technique is that the initial depth map at each viewpoint, obtained through 3D warping operation, is further optimized using segment-based stereo matching.

Camera array pursuits for plenoptic acquisition (CAPPA), [23], have been constructed using Sony XC-333 cameras. It is aimed at capturing multi-view video. Dense and sparse camera arrangement is realized using a modular unit designed for this purpose. Video from 16 cameras is translated to four-screen sequences using four SonyYS-Q430 (quad microprocessor). A final single 16-screen sequence is generated using a fifth quad processor to combines the earlier fourscreen sequences.

Reference [24] has used an array of 64 cameras, Intel Xeon 5160 dual-processor machine, and an NVIDIA GeForce 8800 Ultra graphics card to capture multi-view video in real time. It is also characterized by an interactive control of viewing parameters. 
The International Journal of Multimedia \& Its Applications (IJMA) Vol.6, No.6, December 2014

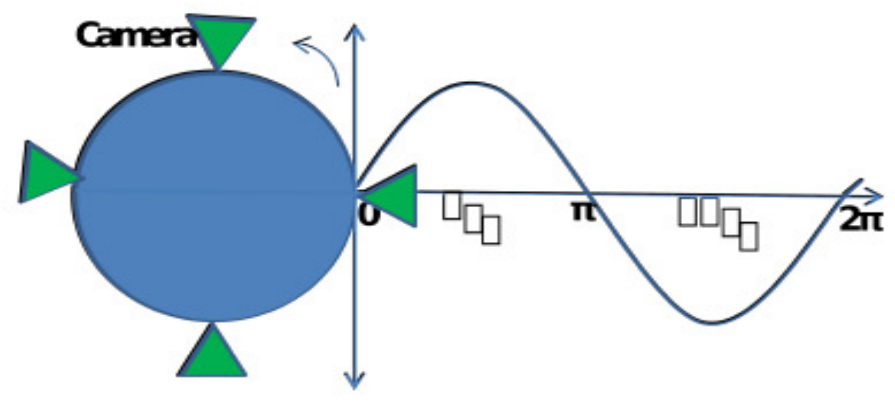

Figure 4. Relating Camera rotation to a sinusoid

Sunex DSL841B lenses with a focal length of 6.1 mm and Marshall Electronics V-4350-2.5 lenses were used in a linear array of cameras to capture indoor and outdoor scenes respectively. In two separate experiments 128 and 48 camera systems were used. Considerable implementation performance and improved image quality have been demonstrated through this experimental setup.

In the work for which [25] is famous, video light fields, high-dynamic-range video, high resolution panoramas, and ultrahigh speed video were generated. In the particular cases of high dynamic range video, high resolution panoramas, and ultrahigh speed video, varying of exposure times, splaying of direction of view, and staggering of camera triggering times were respectively performed.

\subsection{Convergence Array}

It is interestingly observed that the convergence camera arrangement provides detailed information about a scene or an object. Convergence camera configuration has been used in the experimental system of FVT, [26] in order to acquire high-resolution video and analogue signal up to $96 \mathrm{kHz}$. It is a "100-camera system" JAI PULNiX TM-1400CL developed at Nagoya University and Tanimoto Laboratory.

In another experiment, [27], a stadium is surrounded with eight texture acquisition video cameras (SONY DXC-9000) which are capable of performing a progressive scan. A similar camera positioned on the stadium ceiling acquires the $\mathrm{Z}$ component of a player's position. In this experimentation, the fields of view (FOV) of the horizontal plane cameras are controlled by the FOV of the single vertical plane camera. This is done to extend the FOV of the multiple cameras to the stadium areas that were not initially covered.

\subsection{Divergence Array}

The simplest divergent camera configuration consists of a camera usually panned horizontally or vertically at certain intervals through an angle of $360^{\circ}$ [28]. Each image sample contains both the geometric and optical properties of different parts of the scene. A panoramic view of the scene is finally constructed by the method of image stitching.

In image capture to be used for concentric mosaicking, cameras are equally spaced out on the circumference of concentric circles. Therefore, all the cameras not only have the same centre of 
The International Journal of Multimedia \& Its Applications (IJMA) Vol.6, No.6, December 2014

projection, [29], but all input image rays are naturally indexed in radius, rotation angle, and vertical elevation.

\section{THE PROPOSED METHOD}

Linear, divergence, and convergence camera arrangements have been used for acquiring visual content in multi-view video. One feature which is common to these camera configurations is that they are planar. All the cameras are constrained to the same, usually horizontal plane. We now explore the design space of trapezoid graph to seek the possibility of having some of the cameras positioned at a different vertical coordinate level.

The strong point of TCA is that it allows for adaptive camera topology by which points within the scene, especially the occluded ones can be optically and geometrically viewed from several different viewpoints either on the edge of the trapezoid or inside it.

\subsection{Trapezoid graph and Maximal Independent Sets}

In a greater generality, in [8] [9], [10], [11], a trapezoid can be obtained from a trapezoid graph, which is an undirected graph $G(V, E)$ consisting of a set of vertices $V=\left\{V_{1}, V_{2}, V_{3}, \ldots V_{n}\right\}$ and a set of edges $E=\left\{e_{1}, e_{2}, e_{3}, \ldots ., e_{n}\right\}$ if and only if $\left(V_{i}, V_{j}\right) \in E$. The concept of MIS allows for the determination of a corner camera viewpoint with maximum cardinality and to be used in conjunction with the initially chosen corner viewpoint on the perimeter of the trapezoid. Using this concept it is possible to determine any two cameras located on the corner points of the trapezoid that can be considered for stereoscopic analysis.

According to the algorithm discussed in [32] and depicted in Figure 5, MIS can be computed by using as input a trapezoid graph, with its trapezoid representation. The following steps will then be implemented: Step 1: Compute the set of all plane points S. Step 2: Compute Xi, Di. Step 3: Construct the tree T. Step 4: Compute all the paths from leaf nodes to the root of the tree T, i.e., the array path. Step 5: Identify each maximal independent set from the array path. All maximal independent sets are then generated as output.
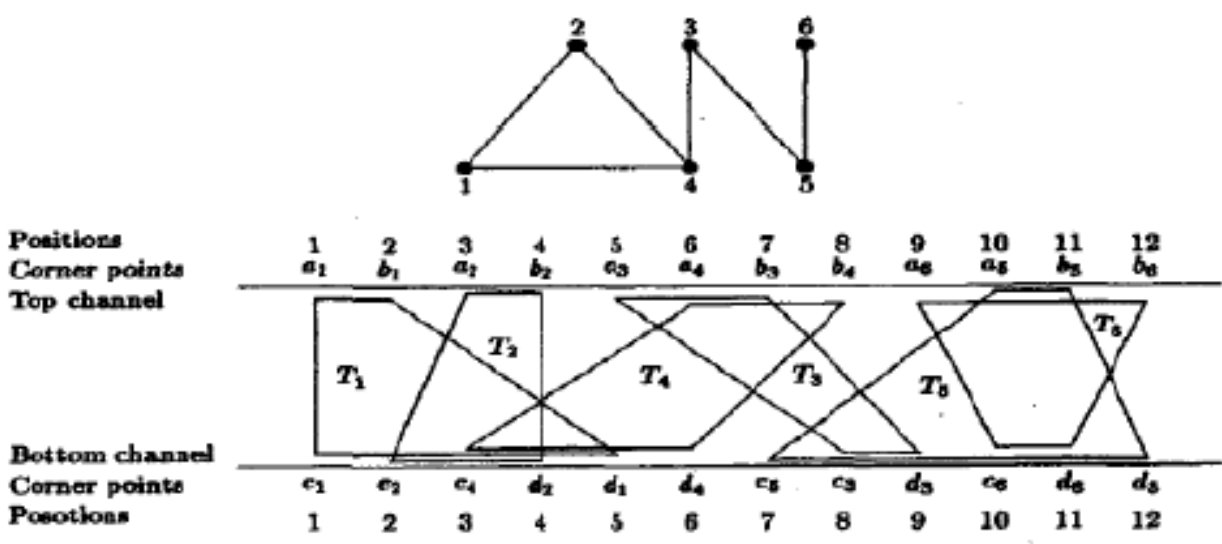

Figure 5. Trapezoid graph and its representation 
The International Journal of Multimedia \& Its Applications (IJMA) Vol.6, No.6, December 2014

\subsection{Formal Mathematical Statement of TCA}

A trapezoid is considered based on the inclusive definition of having high significance in mathematical analysis. It is well known that any proven property of a trapezoid automatically holds for a rectangle, square, rhombi, parallelogram. Mathematically speaking, a quadrilateral in Figure 6 can be described using the relationship in (1).

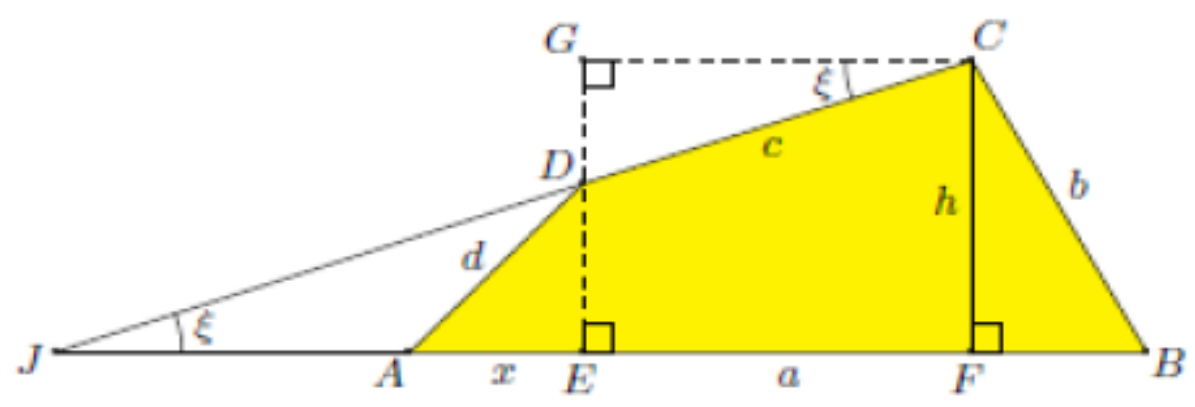

Figure 6. Alternate angle definition of quadrilateral

$$
(\mathrm{AC})^{2}+(\mathrm{BD})^{2}=(\mathrm{BC})^{2}+(\mathrm{AD})^{2}+2(\mathrm{AC})(\mathrm{CD}) \cos \xi
$$

Such a quadrilateral simplifies to a trapezoid, [17], when $\xi$ equals zero as can be seen in Figure 6. This means CD is parallel to AC. This is the definition of a trapezoid that is adopted in this work.

Investigating the base width of a given trapezoid of height $\mathrm{N}$ shown in Figure 7 can provide useful information as to how the stereoscopic distance AD can be computed. The perimeter length, $\mathrm{L}$ of the upper-half hexagon is given as

$$
L=l_{2}+2 \mathrm{~N}\left(\frac{w^{2}}{N^{2}}+1\right)^{\frac{1}{2}}
$$

If the cross-sectional area of the trapezoid is $\mathrm{C}_{\mathrm{a}}$ then

$$
l_{2}=\frac{C_{a}}{N}-N\left(\frac{w}{N}\right)
$$

The $\mathrm{N}$ rate of change of $\mathrm{L}$ can be expressed as

$$
\frac{\partial L}{\partial N}=-C_{a} N^{-2}+N\left(\frac{w}{N^{2}}\right)-\frac{w}{N}+2\left(\frac{w^{2}}{N^{2}}+1\right)^{\frac{1}{2}}+2 N\left(\frac{1}{2}\right)\left(\frac{w^{2}}{N^{2}}+1\right)^{\frac{-1}{2}}\left(-2 \frac{w^{2}}{N^{3}}\right)
$$

By taking the maximum of (4) into consideration, the allowable cross-sectional area, $\mathrm{C}_{\mathrm{a}}$ of the trapezoid can be obtained as in (5). 
The International Journal of Multimedia \& Its Applications (IJMA) Vol.6, No.6, December 2014

$$
C_{a}=N^{2}\left[2\left(\frac{w^{2}}{N^{2}}+1\right)^{\frac{1}{2}}-\left(\frac{w^{2}}{N^{2}}+1\right)^{\frac{-1}{2}}\left(2 \frac{w^{2}}{N^{2}}\right)\right]
$$

In a special case where $\mathrm{w}$ is small compared with $\mathrm{N}, \mathrm{C}_{\mathrm{a}}$ reduces to

$$
\mathrm{C}_{\mathrm{a}}=2 \mathrm{~N}^{2}
$$

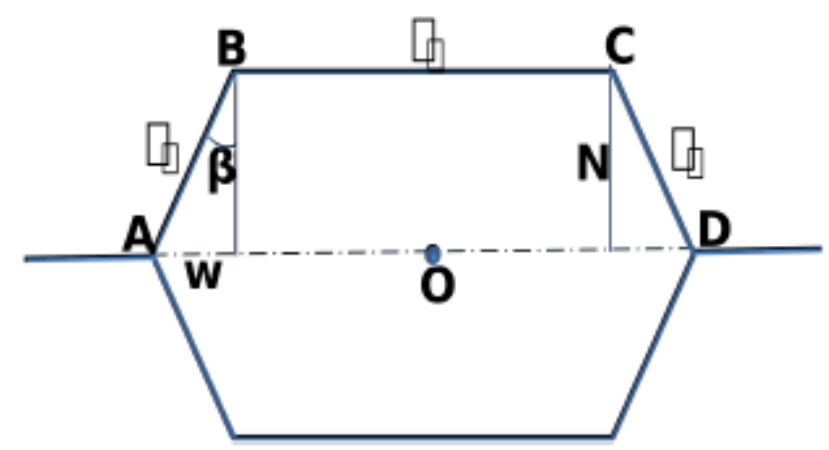

Figure 7. Trapezoidal representation

The expression in (6) implies that the area of the trapezoid is approximately twice the area of a square of side $\mathrm{N}$.

When two cameras were positioned at $\mathrm{A}$ and $\mathrm{D}$, the stereoscopic distance $\mathrm{AD}$, is given by (7). The simplification of (7) indicates that $\mathrm{AD}$ equals twice $l_{1}$. This is a unique property of a trapezoid which expresses the distance between the bottom parts of the legs of a trapezoid as a function of trapezoid sides.

$$
A D=N\left[2\left(\frac{w^{2}}{N^{2}}+1\right)^{\frac{1}{2}}-\left(\frac{w^{2}}{N^{2}}+1\right)^{\frac{-1}{2}}\left(\frac{w^{2}}{N^{2}}\right)\right]+w
$$

Therefore, the stereoscopic distance for any two cameras positioned along the base AD of the trapezoid can always be determined very easily.

Also the perimeter length $\mathrm{L}$ of the trapezoid is described as

$$
L=4 N\left(\frac{w^{2}}{N^{2}}+1\right)^{\frac{1}{2}}-\left(\frac{w^{2}}{N^{2}}+1\right)^{\frac{-1}{2}}\left(\frac{w^{2}}{N^{2}}\right)
$$

Based on a similar analysis, the $\beta$ rate of change of $L$ simplifies to (9). It is clear from (9) that cameras positioned on the corner points of a trapezoid can be made to have equal stereoscopic distance when the trapezoid is half-hexagon in which all the sides are equal. This observation provides a unique way to virtually increase camera density. 


$$
\sin \beta=\frac{1}{2}
$$

\subsection{Characterization of Trapezoid}

A trapezoid has five important characteristics which can be explored in the computation of camera positions in TCA. These are sides and distances, collinearity, trigonometry, area [16] and v-parallelogram characteristics.

\subsubsection{Sides and Distances}

In the trapezoid of Figure 7, triangles $\mathrm{ACB}$ and $\mathrm{DBC}$ have equal areas since they both have an altitude of $\mathrm{N}$. Therefore, it can be written that

$$
\frac{1}{2}\left(l_{2}\right)\left(\sin \left(\frac{\pi}{2}-\beta\right)\right) l_{1}=\frac{1}{2}\left(l_{2}\right)\left(\sin \left(\frac{\pi}{2}-\beta\right)\right) l_{3}
$$

The distance $\mathrm{w}$ can be obtained by equating the sum of the left and right hand-terms of (10) with (8). In this way, the coordinate description of the position of any camera located along $\mathrm{AB}$ can be easily obtained. Also, in the event of any cameras located at the point of intersection of diagonals $\mathrm{AC}$ and $\mathrm{BD}$, the position coordinates of any such cameras can be determined based on the generalized Euler parallelogram law expressed in (11). $\mathrm{X}$ is the horizontal difference between the center point of AC and D. $X=\frac{(A D-B C)}{2}$ In certain specialized applications, cameras positioned at the midpoints of the trapezoid diagonals can also be explored to attempt to solve the problem of occlusion.

$$
(\mathrm{AB})^{2}+(\mathrm{BC})^{2}+(\mathrm{CD})^{2}+(\mathrm{AD})^{2}=(\mathrm{AC})^{2}+(\mathrm{BD})^{2}+4(\mathrm{X})^{2}
$$

For any two cameras positioned at centers of $\mathrm{AB}$ and $\mathrm{CD}$, the stereoscopic distance $\mathrm{SD}$, is computed as

$$
S D=\frac{1}{2}(B C+A D)
$$

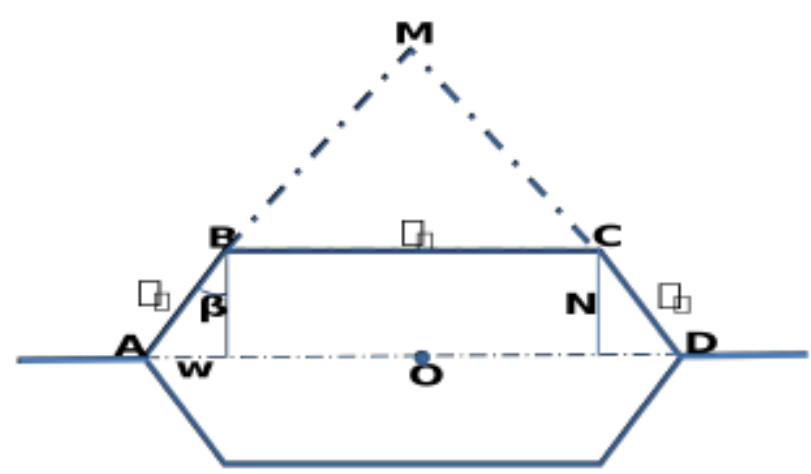

Figure 8. Trapezoid representation 
Assuming two cameras are positioned at $L$ and $L^{\prime}$ on the non-parallel sides AB and CD of the trapezoid of Fig. 7. Let $\mathrm{k}$ be the same ratio by which $L$ and $L^{\prime}$ divide the non-parallel sides. The mathematical analysis provided by [12] shows that the stereoscopic distance $L L^{\prime}$ can be obtained as

$$
L L^{\prime}=k(1-k) B C+k A D
$$

The dependency of the diagonals on the sides and $\beta$ can be formulated for an efficient trapezoid (all sides are equal) as

$$
\begin{gathered}
(A C)^{2}=N^{2}+\left(w+l_{2}\right)^{2} \\
(B D)^{2}=N^{2}+\left(w+l_{2}\right)^{2} \\
l_{1}^{2}=l_{3}^{2}=w^{2}+N^{2} \\
(A C)^{2}+(B D)^{2}=4 l_{1}^{2}(1+\sin \beta)
\end{gathered}
$$

The relationship in (16) states that for an efficient trapezoid, the sum of the squares of the diagonal is directly proportional to the square of the side length $l_{1}$. The constant of proportionality equals 6 . Again, when $\beta$ is equal to zero degrees, (16) simplifies to the expression expected for the sum of squares of the diagonal of a square. This is a further confirmation of the validity of (16).

In an attempt to solve the conceptual and implementation challenges, the trapezoid of Figure 8 will be used to define a scene. This is will be further explained in section 4 .

\subsubsection{Collinearity}

Consider a circle drawn through the corner points of the two halves of the trapezoids shown in Figure 7. By applying the analysis of Christopher Bradley, [36] as demonstrated in Figure 9, to Figure 7, points similar to E, F, G, and $\mathrm{N}$ which are collinear will be obtained. This means that the point of intersection $\mathrm{E}$ of the diagonals of quadrilateral $\mathrm{ABCD}$, the point of intersection $\mathrm{G}$ of the centroids of triangles $\mathrm{BCD}, \mathrm{ACD}, \mathrm{ABD}, \mathrm{ABC}$, the point of intersection $\mathrm{N}$ of the midpoints of $\mathrm{AB}, \mathrm{BC}, \mathrm{CD}, \mathrm{DA}$ and the point of intersection $\mathrm{F}$ of the centroids of triangles $\mathrm{ABE}, \mathrm{BCE}, \mathrm{CDE}, \mathrm{DAE}$ all lie on a line and hence are collinear. These points are potential candidate camera viewpoints which could be explored in an attempt to challenge the problem of scene point visibility. 


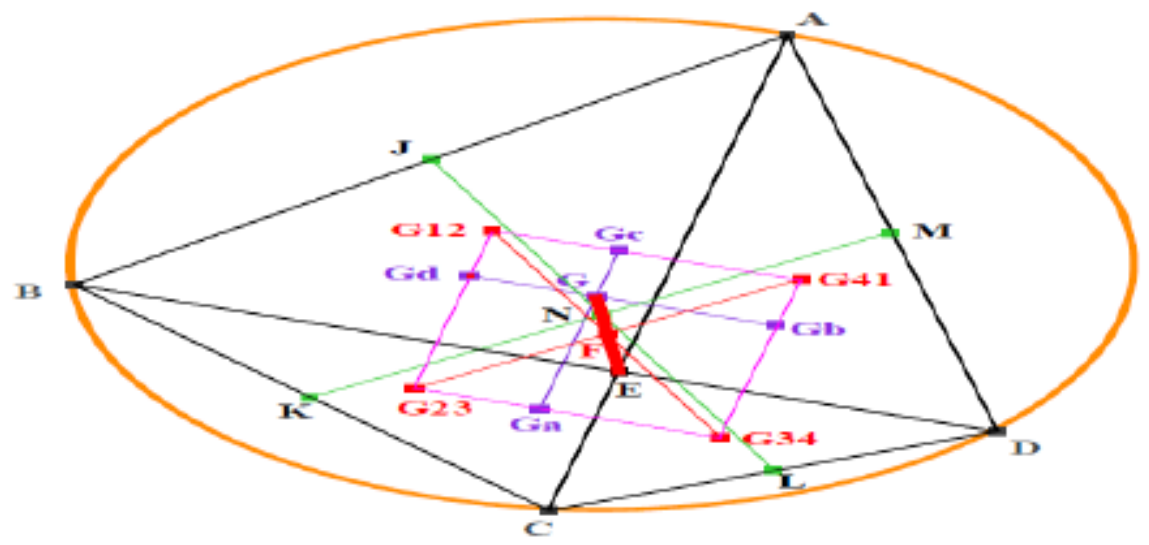

Figure 9. Cyclic quadrilateral

\subsubsection{Area}

The area characteristic of a trapezoid is important in the computation of scene volume. An extension of the work in [13] provides a clue to the area of trapezoid as in (17)

$$
A=\frac{1}{4} \sin \vartheta\left(\left(2\left((A B)^{2}+(C D)^{2}\right)-4 X^{2}\right)\left(2\left(B C^{2}+A D^{2}\right)-4 X^{2}\right)\right)^{\frac{1}{2}}
$$

\subsubsection{Similarity}

Assuming in Fig. 7 that the diagonals $\mathrm{AC}$ and $\mathrm{BD}$ intersect at a point $\mathrm{P}$. Let the perpendicular lines from $P$ to $A D$ and $B C$ have lengths $h 1$ and $h 2$ respectively. Also let $\theta_{1}$ and $\theta_{2}$ respectively be the angles subtended by $\mathrm{AC}$ and $\mathrm{BD}$ with $\mathrm{AD}$. The following two equations can be written

$$
\begin{gathered}
h_{1}=A P \sin \theta_{1}=P D \sin \theta_{2} \\
h_{2}=P C \sin \theta_{1}=P B \sin \theta_{2} \\
\frac{A P}{P C}=\frac{P D}{P B}
\end{gathered}
$$

Expression (20) is a trapezoidal similarity characteristic which can facilitate the computation of the coordinates of any cameras positioned at $\mathrm{P}$.

\subsubsection{V-Parallelogram Characterization}

V-parallelogram characterization of trapezoids has been at the center of recent research investigation in mathematics aimed at solving problems of broad relevance [37],[38]. It is said to be formed when points on any adjacent sides of a trapezoid are joined with a line which is parallel to a diagonal of the trapezoid which does not go through the angle between the adjacent sides and the one opposite it. This definition implies that each corner point of a V-parallelogram 
lie on one and only one side of the trapezoid. Therefore, it means that cameras positioned on adjacent sides of a trapezoid can stereoscopically be used to observe a scene.

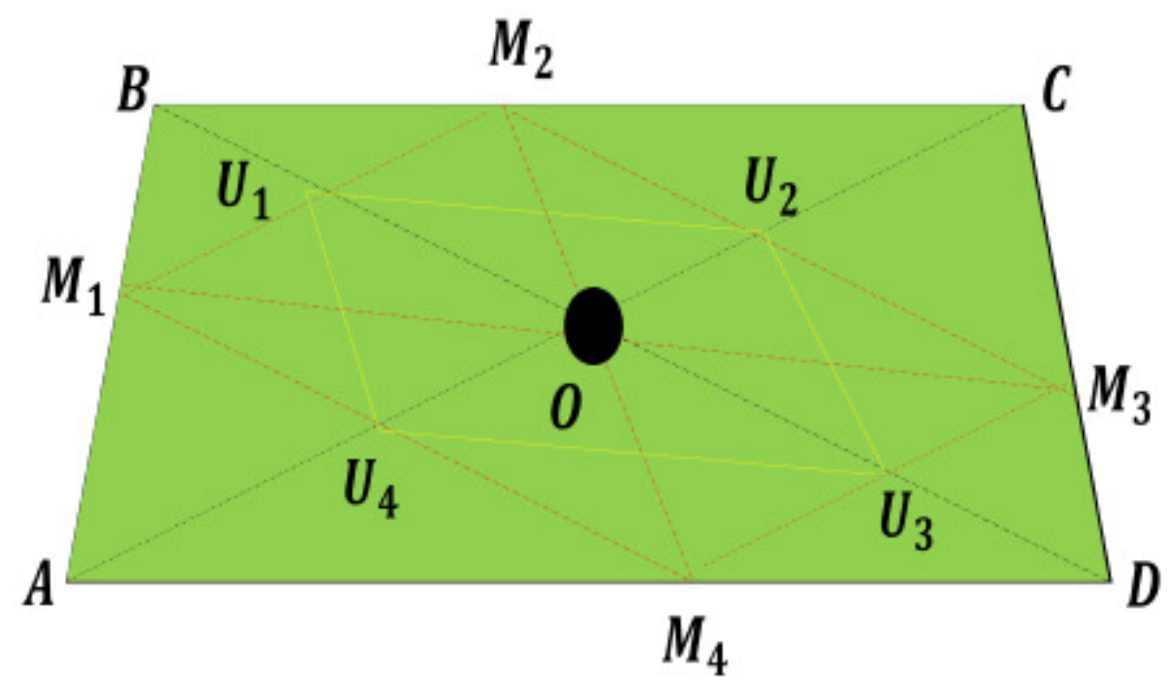

Figure 10. V-parallelogram $\mathrm{M}$ of a trapezoid

In multi-view visual content acquisition, cameras can also be positioned at viewpoints $\mathrm{M}_{1}, \mathrm{M}_{2}$, $\mathrm{M}_{3}, \mathrm{M}_{4}$ which, according to a statement in Euclidean geometry define a v-parallelogram $\mathrm{M}_{1} \mathrm{M}_{2} \mathrm{M}_{3} \mathrm{M}_{4}$ shown in Figure 10. The homothetic transformation of triangles $A B C$ and $\mathrm{M}_{1} \mathrm{BM}_{2}$ around the corner point B of the trapezoid in Figure 10 provides for (21) from which the baseline distance can be determined.

$$
\frac{A M_{1}}{A B}=\frac{C M_{2}}{B C}=\frac{A U_{4}}{A O}
$$

\section{IMPLEMENTATION CONSIDERATIONS}

Of course, TCA is potentially inclined to addressing some of the critical challenges in multiview video acquisition; however its implementation could also be a challenge. Two issues easily come to mind namely the possible curvature of the scene and the frequency of the trapezoid.

For a scene of large volume, the curvature of the opposite and parallel sides of the trapezoid becomes significant and adds up to the complexity of the implementation strategy. Practically, this situation implies that the stereoscopic distance can no longer be measured on a straight line between two camera positions.

Again, for a large scene where a small stereoscopic distance is required between cameras, several cycles of TCA will have to be implemented. This will significantly make the cost ofimplementing this strategy very high. A right balance will have to be struck between small and large scenes on one hand and curvature and frequency of TCA on the other. 
The International Journal of Multimedia \& Its Applications (IJMA) Vol.6, No.6, December 2014

To adequately address these issues, an implementation strategy is proposed based on the assumption that the scene is contained in a pyramidal frustum defined by four trapezoids as shown in Figure. 10.

In this method, only four half-cycles of TCA are required for the entire scene. The acquisition cameras are then placed on the edges of the trapezoid such that the ones along the legs of the trapezoid exhibit convergence topology. The idea of scene volume in this work does not require a strong argument for it to be embraced since it has been mentioned in [16] in a different way though implying the same sense.

The formulation of scene volume is actually a fortification of the basic volume (22) of a frustum. The modification of (22) will take into account the near and far depth parameters of the scene.

$$
V=\frac{\left(h_{2}-h_{1}\right)}{3}\left(A_{1}+\left(A_{1} A_{2}\right)^{\frac{1}{2}}+A_{2}\right)
$$

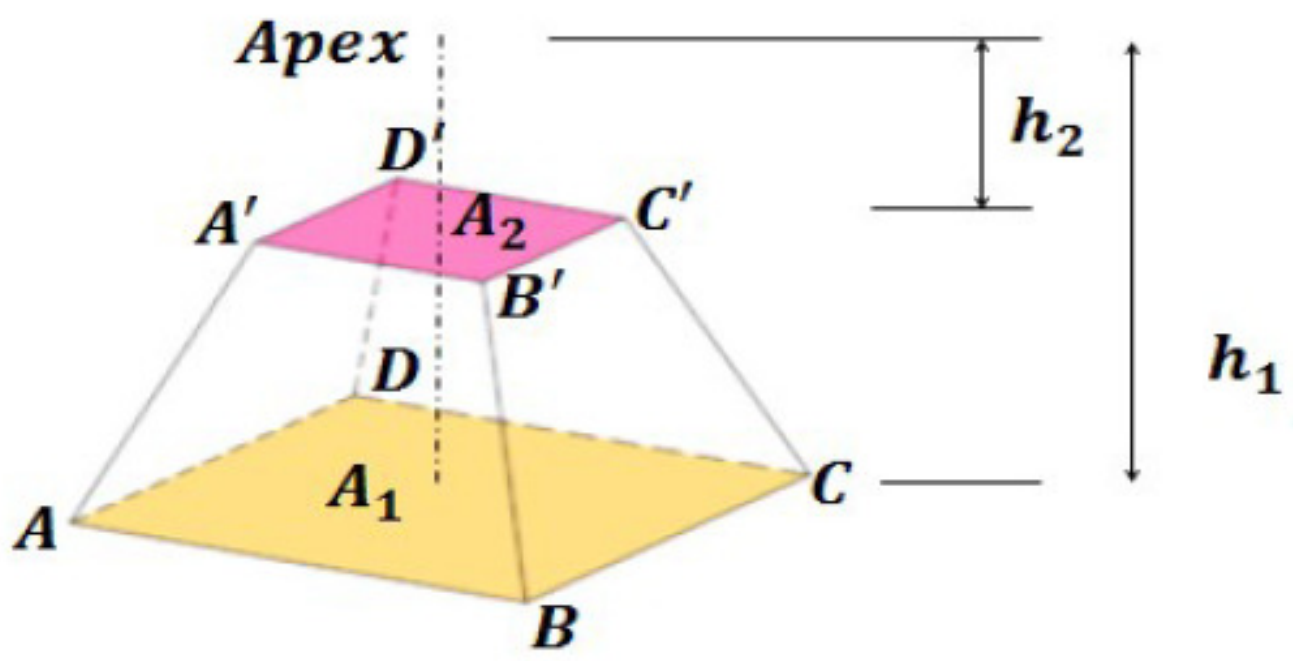

Figure 11. Implementation strategy of TCA

\section{CAMERA AND DEPTH MAP CONSTRUCTION}

Stereoscopic camera system with parallel axes is believed to be necessary to avoid the vertical image disparity generated by systems that verge the camera axes [38]. However, "Two forward facing camera" technique [39] used to deliver a two-dimensional (2D) projection of a scene remains a fundamental challenge in stereo vision. Correspondences between multiple images need to be accurately established. These are understood to be attributable, in the view of [40], to scene properties such as textureless areas, non-Lambertian surfaces, reflections and translucency, and occlusions. Camera issues include image noise, calibration and synchronization, differences in exposure, white balancing, and other radiometric properties.

A camera is formulated based on pin-hole camera model expressed in (23)

$$
\Lambda x=K R T[I \mid-t] \mathrm{X} o=P \mathrm{X}
$$


The International Journal of Multimedia \& Its Applications (IJMA) Vol.6, No.6, December 2014

$\mathrm{K}$ is a $3 \times 3$ matrix consisting of camera intrinsic parameters namely: focal length, aspect ratio, skew and principal point. $\mathrm{R}$ denotes an orthogonal matrix and $\mathrm{t}$ a vector, encoding camera rotation and translation, depth, $\lambda$, is equal to $Z$. Both are determined by the orientation and displacement of the camera at the virtual viewpoint on the edge of the trapezoid. $\mathrm{x}$ and $\mathrm{X}_{0}$ are the extended image and scene point coordinates. The projection matrix $\mathrm{P}$ is used to render the quantization level $\mathrm{v}$, for each vertex and face of the graphic. The substitution of $\mathrm{v}$ into (24) yields the expected depth for all the vertices and faces.

Using uniform quantization of depth value between $\mathrm{Z}_{\text {near }}$ and $\mathrm{Z}_{\text {far }}$ to minimize disocclusion, and taken into consideration the fact that in humans, the perceived depth distance of close objects is much less than the depth distance of further objects [41], [42] depth is formulated as

$$
\text { depth }=\frac{Z_{\text {near }}}{\left(1-\frac{v}{2^{32}}\right)}
$$

where $\mathrm{v}$ is the number of quantization levels from zero through to $2^{32}-1$. The high number of quantization levels is aimed at improving the resolution of the depth map.

\section{EXPERIMENTAL RESULTS}

Some of the simulation results to validate the concept of TCA are shown in Figure 12. Each disparity map of an object is generated using a different virtual viewpoint. These are high quality disparity map. In the analysis leading to the determination of disparity map, correspondence problem still remain a major source of error. This is because correlation based techniques is widely used. It is formulated based on resemblance constraint which requires intensity similarity between any corresponding points [40]. Also, it cannot cope with textureless regions where pixels are insufficiently distinct. Occlusions and discontinuities of some parts of scene are important features which correlation method cannot adequately handle. The choice of window size is critical the regards to accurate disparity map. To reduce the error contribution due to the aforementioned, comparison of quantitative results with Normalized Cross Correlation (NCC) method, Daisy descriptor, Error Quadratic Means (EQM), and Local evidence have been employed in most research work related to depth map.

Since only a single image of the different objects is used in this work, i.e not stereo images, the earlier mentioned objective quality metrics cannot be applied. However, subjective quality assessment (SQA) of the generated disparity maps has been conducted at Centre for Media Communication Research (CMCR), Brunel University, London. Research students who already have a good understanding of disparity map were invited to take a look at the disparity maps for different objects. The SQA was based on double stimulus impairment scale (DSIS) in which the following scales were used. "Very clean and sharp $=5$ ", "Clear but not sharp $=4$ ", "Clear but blur $=3$ ", "blur $=2$ ", and "Not clear $=1$ ". The calculated Mean opinion scores (MOSs) for each disparity map were then analysed. More than 90 percent of the observers scored each disparity map with DSIS of 5. 
The International Journal of Multimedia \& Its Applications (IJMA) Vol.6, No.6, December 2014
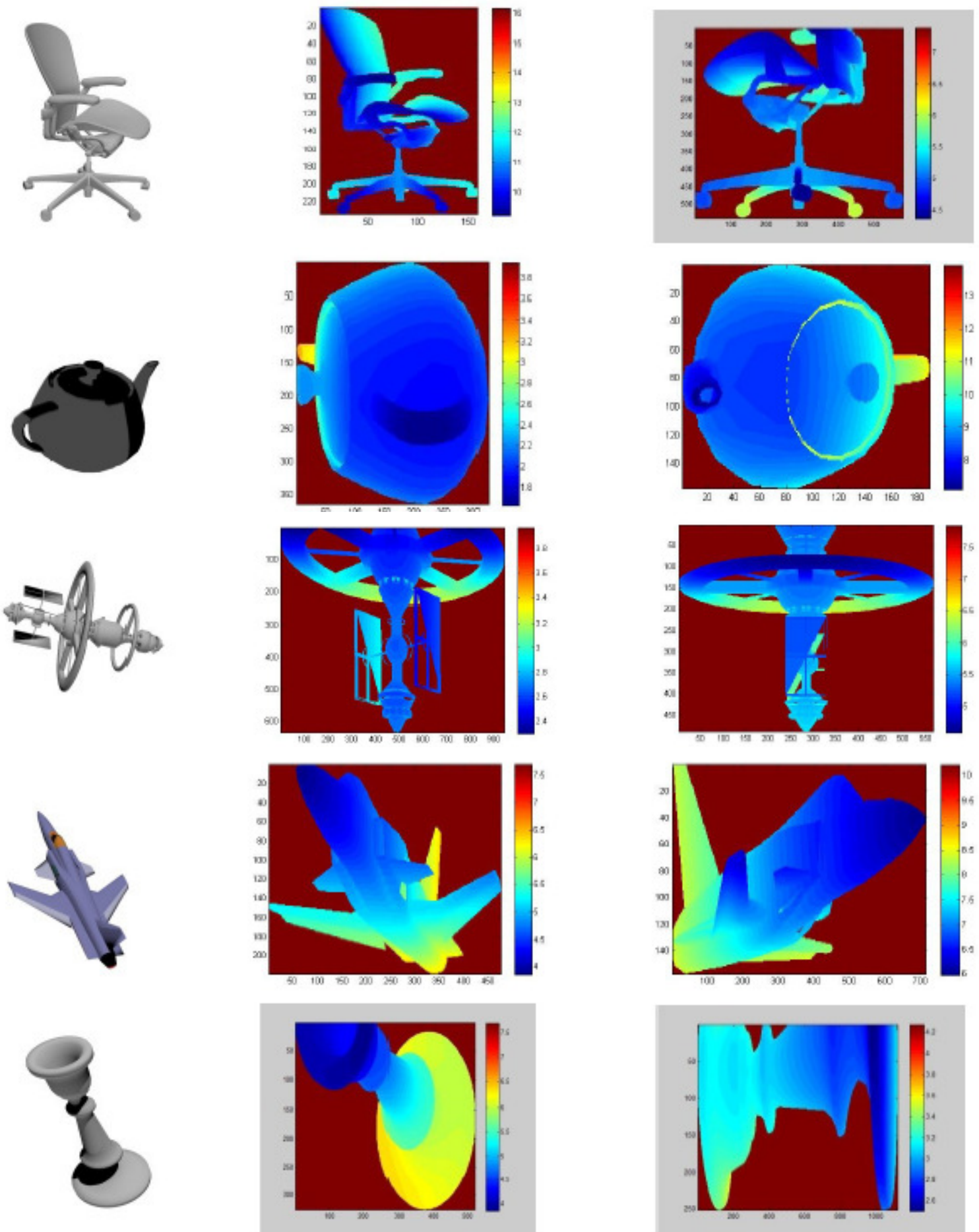

Fig. 12 Objects are shown in the first column. For each object the corresponding disparity maps are given in the same row as the object they represent. 
The International Journal of Multimedia \& Its Applications (IJMA) Vol.6, No.6, December 2014

\section{CONCLUSIONS}

In this paper, the mathematical description of TCA has been presented based on an efficient trapezoid. In principle, the definition of a trapezoid is simple. However, it is the diverse different methods of determining the coordinates of its corner points, points on the edges, and in some critical applications, points in the space defined by the edges that make TCA so appealing in camera positioning for multi-view video. The similarity, sides and distances, areas, and trigonometric characteristics of a trapezoid provide for computation of baseline and coordinate description of camera positions.

Also the understanding provided by TCA has been used for depth map generation with respect to virtual viewpoints on the edge of the trapezoid. Virtual viewpoints which trace a trapezoid guarantee high quality disparity map as indicated by DSIS, with almost complete absence of holes. Therefore, the minimization of deviation from actual disparity which is widely considered as the default measure of success is not required to validate the generated disparity map.

\section{REFERENCES}

[1] V. R Ion and C. Stromeyer, "Affective discrimination of stimuli that cannot be recognised," Science, vol. 207, pp. 1, Feb. 1980.

[2] R. Jain, R. Kasturi and B. G. Schunck, Machine Vision. McGraw-Hill New York, 1995.

[3] P. Merkle, K. Muller and T. Wiegand, "3D video: acquisition, coding, and display," Consumer Electronics, IEEE Transactions on, vol. 56, no. 2, pp. 946-950, May. 2010.

[4] T. Matsuyama, S. Nobuhara, T. Takai and T. Tung, 3D video and its applications. Springer Publishing Company, Incorporated, 2012.

[5] S. Jarusirisawad and H. Saito, "3DTV view generation using uncalibrated pure rotating and zooming cameras," Signal Process Image Commun, vol. 24, pp. 17-30, no. 1, Jan. 2009.

[6] M. Tanimoto, "FTV: Free-viewpoint Television," Signal Process Image Commun, vol. 27, pp. 555-570, Jul. 2012.

[7] A. Kubota, A. Smolic, M. Magnor, M. Tanimoto, C. Zhang, "Multiview imaging and 3DTV," IEEE Signal Process. Mag., vol. 1053, no. 5888/07, pp. 10-21, 2007.

[8] Y. Fu, Y. Guo, Y. Zhu, F. Liu, C. Song and Z. Zhou, "Multi-view video summarization," Multimedia, IEEE Transactions on, vol. 12, no. 7, pp. 717-729, 2010.

[9] L. Onural, 3D Video Technologies : An Overview of Research Trends. Bellingham, Wash: SPIE, 2010, pp. 27-32.

[10] H. K. Aghajan, A. Cavallaro and ScienceDirect (Online service), Multi-Camera Networks Electronic Resource : Principles and Applications. Amsterdam ; Boston: Elsevier, AP, 2009, pp. 29-75.

[11] A. Saxena, J. Schulte and A. Y. Ng, "Depth estimation using monocular and stereo cues." in IJCAI, 2007, pp. 2197-2203.

[12] A. V. Bhavsar and A. N. Rajagopalan, "Towards unrestrained depth inference with coherent occlusion filling," Int J Comput Vision, vol. 97, no. 2, pp. 167-190, 2012.

[13] Q. Huynh-Thu and L. Schiatti, "Examination of 3D visual attention in stereoscopic video content," in IS\&T/SPIE Electronic Imaging, 2011, pp. 78650J-78650J.

[14] M. Lang, A. Hornung, O. Wang, S. Poulakos, A. Smolic and M. Gross, "Nonlinear disparity mapping for stereoscopic 3D," ACM Transactions on Graphics (TOG), vol. 29, no. 4, pp. 75:1$10,2010$.

[15] H. Li, J. Seo, K. Kham and S. Lee, "Measurement of 3D visual fatigue using event-related potential (ERP): 3D oddball paradigm," in 3DTV Conference: The True Vision-Capture, Transmission and Display of 3D Video, 2008, 2008, pp. 213-216. 
The International Journal of Multimedia \& Its Applications (IJMA) Vol.6, No.6, December 2014

[16] D. Chrysostomou and A. Gasteratos, "Optimum multi-camera arrangement using a bee colony algorithm," in Imaging Systems and Techniques (IST), 2012 IEEE International Conference on, 2012, pp. 387-392.

[17] R. Schneiders, "Algorithms for quadrilateral and hexahedral mesh generation," Proceedings of the VKI Lecture Series on Computational Fluid Cynamics, 2000.

[18] M. Josefsson, "Characterizations of trapezoids," in Forum Geometricorum, 2013, pp. 23-35.

[19] V. Vaish, M. Levoy, R. Szeliski, C. L. Zitnick and S. B. Kang, "Reconstructing occluded surfaces using synthetic apertures: Stereo, focus and robust measures," in Computer Vision and Pattern Recognition, 2006 IEEE Computer Society Conference on, 2006, pp. 2331-2338.

[20] N. A. Dodgson, "Resampling radially captured images for perspectively correct stereoscopic display," in Photonics West'98 Electronic Imaging, 1998, pp. 100-110.

[21] W. Jang and Y. Ho, "Direct Depth Value Extraction Method for Various Stereo Camera Arrangements," in The 2013 International Conference on Embedded System and Intelligent Technology (ICESIT 2013), 2013, pp. 128-131.

[22] Y. Ho, "Challenging Technical Issues of 3D Video Processing," Journal of Convergence, vol. 4, no. 1, pp. 1-6, Mar. 2013.

[23] E. K. Lee and Y. S. Ho, "Generation of high-quality depth maps using hybrid camera system for 3-D video," Journal of Visual Communication and Image Representation, vol. 22, no. 1, pp. 7384, Jan. 2011.

[24] T. Naemura, J. Tago and H. Harashima, "Real-time video-based modeling and rendering of 3D scenes," Computer Graphics and Applications, IEEE, vol. 22, no. 2, pp. 66-73, Mar. 2002.

[25] Y. Taguchi, T. Koike, K. Takahashi and T. Naemura, "TransCAIP: A live 3D TV system using a camera array and an integral photography display with interactive control of viewing parameters," Visualization and Computer Graphics, IEEE Transactions on, vol. 15, no. 5, pp. 841-852, Sep. 2009.

[26] M. Levoy, "Light fields and computational imaging," Computer, vol. 39, no. 8, pp. 46-55, Aug. 2006.

[27] M. Tanimoto, "Overview of free viewpoint television," Signal Process Image Commun, vol. 21, no. 6, pp. 454-461, 07, Jul. 2006.

[28] T. Koyama, I. Kitahara and Y. Ohta, "Live mixed-reality 3d video in soccer stadium," in Mixed and Augmented Reality, 2003. Proceedings. The Second IEEE and ACM International Symposium on, 2003, pp. 178-186.

[29] M. Brown and D. G. Lowe, "Automatic panoramic image stitching using invariant features," International Journal of Computer Vision, vol. 74, no. 1, pp. 59-73, 2007.

[30] M. Gong and Yee-Hong Yang, "Rayset: a Taxonomy for Image-Based Rendering," International Journal of Image \& Graphics, vol. 6, no. 3, pp. 313-339, Jul. 2006.

[31] I. Dagan, M. C. Golumbic and R. Y. Pinter, "Trapezoid graphs and their coloring," Discrete Applied Mathematics, vol. 21, no. 1, pp. 35-46, Sep. 1988.

[32] M. Hota, M. Pal and T. K. Pal, "An efficient algorithm to generate all maximal independent sets on trapezoid graphs," International Journal of Computer Mathematics, vol. 70, no. 4, pp. 587599, 1999.

[33] C. Flotow, "On powers of m-trapezoid graphs," Discrete Applied Mathematics, vol. 63, no. 2, pp. 187-192, Nov. 1995.

[34] G. B. Mertzios and D. G. Corneil, "Vertex splitting and the recognition of trapezoid graphs," Discrete Applied Mathematics, vol. 159, no. 11, pp. 1131-1147, Jul. 2011.

[35] L. Droussent, "On a theorem of J. Griffiths," The American Mathematical Monthly, vol. 54, no.9, pp. 538-540, Nov. 1947.

[36] C. Bradley, Three centroids created by a cyclic quadrilateral, in: Article: CJB/2011/141, 2011, pp. 1-3.

[37] M. Josefsson, "Five proofs of an area characterization of rectangles," in Forum Geometricorum, 2013, pp. 17-21.

[38] M. F. Mammana, B. Micale and M. Pennisi, "Properties of valtitudes and vaxes of a convex quadrilateral," in Forum Geometricorum, 2012, pp. 47-61. 
The International Journal of Multimedia \& Its Applications (IJMA) Vol.6, No.6, December 2014

[39] G. R. Jones, D. Lee, N. S. Holliman and D. Ezra, "Controlling perceived depth in stereoscopic images," in Photonics West 2001-Electronic Imaging, 2001, pp. 42-53.

[40] H. Fradi and J. Dugelay, "Improved depth map estimation in stereo vision," in IS\&T/SPIE Electronic Imaging, 2011, pp. 78631U-78631U-7.

[41] C. L. Zitnick and S. B. Kang, "Stereo for image-based rendering using image over segmentation," International Journal of Computer Vision, vol. 75, pp. 49-65, 2007.

[42] Yu-Cheng Fan and Tsung-Chen Chi, "The novel non-hole-filling approach of depth image based rendering," in 2008, pp. 325-328. 